\title{
Legitimationsmythen des deutschen Theaters: eine institutionsgeschichtliche Perspektive
}

\section{Christopher Balme}

\section{Zusammenfassung}

Dieser Aufsatz untersucht die gegenwärtigen Krisendiskurse unter dem Blickwinkel des Begriffs der Legitimation aus historischer Perspektive. Legitimationsmythen entstehen der neoinstitutionalistischen Theorie zufolge, wenn Institutionen unter Druck geraten und versuchen, sich als zentral für die kulturellen Traditionen ihrer Gesellschaften zu etablieren, um offiziellen Schutz zu erhalten. Der Aufsatz diskutiert mehrere solche Mythen in Kategorien von „Gütern“, wie z. B. Theater als moralisches, gemeinschaftliches oder als öffentliches Gut.

\section{Schlüsselwörter}

Legitimation • Theater • Neoinstitutionalismus

\section{$1 \quad$ Einführung}

Die bemerkenswerte und im internationalen Vergleich immer wieder bewunderte künstlerische Vielfalt der deutschsprachigen Theater und Orchester wird gegenwärtig überlagert von Unsicherheit über ihre tatsächliche gesellschaftliche Wertschätzung und ihr Zukunftspotential. Man spricht regelrecht von einer ,Krise“ (Schmidt 2017), die sich jedoch auf recht widersprüchliche Art und Weise

C. Balme $(\varangle)$

Institut für Theaterwissenschaft, Ludwig-Maximilians-Universität München, München, Deutschland

E-Mail: balme@lmu.de 
manifestiert: Zum einen werden unter Sparzwängen der deutschen Kulturpolitik Theater und Orchester fusioniert oder ganze Sparten geschlossen, zum anderen wird das System jenseits rein ökonomischer Zwänge von Interessenverbänden und Medien selbst dort infrage gestellt, wo kein Besuchsrückgang, sondern verfestigte Publikumsstrukturen zu verzeichnen sind. Die erfolglose deutsche Bewerbung 2019 um Aufnahme in die repräsentative Liste des Immateriellen Kulturerbes der Menschheit der UNESCO zeugt von einer solchen Unsicherheit und wachsendem Krisenbewusstsein, da die Bewerbung durch die mächtigsten Verbände des Theater- und Musiklebens, den Deutschen Bühnenverein und den Deutschen Musikrat, unterstützt vom Dachverband der Freien Darstellenden Künste vorangetrieben wurde. Solche Symptome deuten darauf hin, dass die Legitimität des ganzen Systems, manchmal auch von den Akteuren selbst infrage gestellt wird. Wenn von der Theaterwissenschaft der wohl wichtigste Grundpfeiler des Systems, das Stadttheater, als „Mythos“ apostrophiert (Roselt 2013, S. 217) wird, so ist es naheliegend, die beiden Kernbegriffe „Legitimation“ und „Mythos“ institutionstheoretisch und -geschichtlich unter die Lupe zu nehmen. ${ }^{1}$

Im vorliegenden Aufsatz wird der Versuch unternommen, die gegenwärtigen Krisendiskurse historisch zu perspektivieren, vor allem unter dem Blickwinkel des Begriffs der Legitimation. Wenn wir heute von einer Legitimationskrise sprechen, von den Autoren des Kulturinfarkts polemisch als ,"Von allem zuviel und überall das Gleiche“ (Haselbach et al. 2012) formuliert, müssen wir uns vergegenwärtigen, dass die heutige(n) Legitimationskrise(n) historische Wurzel haben, die teilweise in eine ,mythische“ Urzeit zurückreichen.

\section{$2 \quad$ Legitimation und Neoinstitutionalismus}

In den letzten drei Jahrzehnten hat eine Revolution in der Institutionentheorie stattgefunden, die gewöhnlich unter dem Etikett des „Neoinstitutionalismus“ subsumiert wird (DiMaggio und Powell 1991; Scott 2013; Walgenbach und Meyer 2008). Diese Neubestimmung der Beziehung zwischen Institutionen und Organisationen hat einen viel flexibleren Rahmen für das Verständnis der Auswirkungen von Institutionen auf unser tägliches Leben geschaffen und gezeigt, dass es nur wenige Bereiche, einschließlich des Theaters, gibt, die nicht in irgendeiner Weise

\footnotetext{
${ }^{1}$ Bei Roselt heißt es: „Es wird damit behauptet, dass die hier skizzierte Idee des Stadttheaters ein Mythos ist, der als solcher erst durch nachträgliche Erzählungen entstanden ist, und wie jeder Mythos - seine Wirkmacht unabhängig von seiner historischen Verifizierbarkeit entfaltet“" (2013, S. 217).
} 
durch institutionelle Strukturen bestimmt sind, auch wenn die Bedeutung von normativen Regeln und Routinen sehr unterschiedlich bewertet wird (Meyer et al. 2009, S. 40). Neoinstitutionelle Ansätze neigen dazu, eine klare Unterscheidung zwischen den Ebenen von Institution und Organisation zu treffen. Erstere bezieht sich auf abstrakte Regeln und Rahmen, die sich in den letzteren, individuellen Organisationen, manifestieren, wobei die beiden Ebenen durch wechselseitige Beziehungen miteinander verbunden sind. Schließlich versucht der Neoinstitutionalismus zu erklären, wie Organisationen sich selbst perpetuieren, indem sie Mythen inkorporieren, die sich als unhinterfragte institutionelle Regeln verstehen.

In Anlehnung an die berühmte Definition von Douglass North (1990) können wir sagen, dass Institutionen ,die Spielregeln“ definieren und die von Menschen erdachten Zwänge darstellen, die die menschliche Interaktion formen (1990, S. 3), wobei Organisationen - ,groups of individuals bound by some common purpose to achieve objectives" (1990, S. 5) - die Akteure sind. Theaterwissenschaftler*innen neigen dazu, sich mit der Organisationsebene, den Akteuren - bestimmten Künstler*innen, Theatern und Theatergruppen - zu beschäftigen, denn hier wird Theater hergestellt und sichtbar gemacht. Weniger sichtbar ist die institutionelle Ebene, die Spielregeln, die in den meisten Fällen eine Form exogener Unterstützung beinhaltet, sei es durch ein Ministerium für Kultur, öffentlich finanzierte Universitäten oder durch private Förderung, wie etwa transnational operierende Philanthropie. All diese Fälle schaffen Regeln und Zwänge, die in hohem Maße wandelbar sind. Durch Gesetzesänderungen, neue Förderschwerpunkte oder Debatten in der Öffentlichkeit kann sich der institutionelle Rahmen ändern. In der Interaktion zwischen der institutionellen und der organisatorischen Ebene entstehen Strukturen, die der Theateranalyse zugänglich sind.

Hier von Interesse ist die institutionelle Ebene, die Spielregeln, die bestimmen, wie Organisationen sich verhalten. Die Art und Weise, wie sich Organisationen Anerkennung und Macht aneignen und sichern, gehört zu den zentralen Erkenntnissen der Institutionentheorie. Institutionen wiederum entstehen und verfestigen sich durch Legitimationsprozesse, ob sie juristisch sanktioniert, moralisch autorisiert oder als Bestandteil geteilter kultureller und kognitiver Rahmen sind (Scott 2013). Dass das deutsche Stadt- und Staatstheater-System Ergebnis eines solchen Prozesses ist, kann als gesichert gelten. Es handelt sich um selbstreproduzierende, selten hinterfragte, weil in Praktiken, Vorstellungen und Mythen eingebettete Regelsysteme.

Legitimation spielt auch in der älteren Institutionsforschung eine wichtige Rolle, wie z. B. in Max Webers (2002) Überlegungen zum sozialen Handeln, wo er „Legitimitäts-Glauben“ entlang eines Kontinuums zwischen ,bloß traditional oder bloß zweckrational motivierter Orientierung an einer Ordnung“ 
akzeptiert (2002, S. 683). Auch in seiner Typologie der "legitimen Herrschaft“ umfasst Legitimierung sowohl Tradierung als auch legal-bürokratische Formen mit Satzung sowie charismatische Ausprägungen dort, wo Legitimierung über affektuell-rituellen Glauben erfolgt. Die Dimension der Legitimation in der neueren neoinstitutionalistischen Forschung unterscheidet sich von der älteren Tradition vor allem dadurch, dass sie diese Dimension als in hohem Maße konstruiert (Meyer et al. 2009, S. 41) betrachtet.

Theaterhistorisch ist der Begriff der Legitimität bereits in England im 18. Jahrhundert verbürgt, wo die Unterscheidung zwischen legitimate und illegitimate drama sowohl auf den Status der lizenzierten Theater als auch auf die dort zulässigen Theatergattungen hinweist. Legitime Theater waren diejenigen, die über ein königliches Patent verfügten - eigentlich nur Covent Garden und Drury Lane und damit das Recht hatten, reines Sprechtheater (legitimate drama) aufzuführen. Andere Bühnen etablierten sich in den rechtlichen Zwischenräumen des Illegitimen (aber nicht notwendigerweise Illegalen) und entwickelten musikalische Zwischengattungen wie Vaudevilles, Harlekinaden, Melodrama und Singspiele (Balme 2017).

Nach der neoinstitutionellen Theorie wird, wie oben ausgeführt, das Verhältnis zwischen Institutionen und den Gesellschaften, in denen sie sich befinden, durch den Wunsch und das Bedürfnis nach Legitimität bestimmt: „Legitimacy is perhaps the most central concept in institutional research", so die Soziolog*innen Jeanette Colyvas und William Powell (2006, S. 308). Legitimität ist eine Wahrnehmung oder Annahme, dass die Handlungen einer Organisation oder Institution wünschenswert, richtig oder angemessen sind und diese innerhalb eines gesellschaftlich konstruierten Systems von Normen, Werten, Überzeugungen und Definitionen ihre Grundlage haben. Diese „kulturell-kognitive Säule“ (Scott 2013, S. 66) von Institutionen bedeutet, dass solche kognitiven Rahmen oft wichtiger sind als normative, wie etwa Gesetze, was sie für Veränderungen sehr viel förderlicher macht. Ein Schlüsselindikator für Institutionalisierung ist der Status des „taken-for-grantedness“, auf dessen Basis also etwas unhinterfragt akzeptiert wird.

Der Idealzustand des ,taken-for-grantedness“ wird durch Mythen gestützt, wie Meyer und Rowan (1977) in einem wegweisenden Essay argumentieren. Mythen sind die Geschichten, die wir uns über uns selbst erzählen: In institutionelle Begrifflichkeit übersetzt, bedeutet das nicht, dass sie unwahr sind, nur dass sie normalerweise nicht infrage gestellt, sondern eher akzeptiert und verteidigt werden, insbesondere in Zeiten der Instabilität und des Wandels: „Organizations under attack in competitive environments (...) attempt to establish themselves as central to the cultural traditions of their societies in order to receive official protection“ (Meyer und Rowan 1977, S. 348). Hier beziehen sich die Autoren 
nicht auf das deutsche Stadt- und Staatstheater, sondern auf kleine Bauernhöfe, öffentliche Eisenbahnen und das damals kriselnde Unternehmen Rolls-Royce.

Das Theater hat jedoch sogar einen höheren Anspruch auf Anerkennung als „,central to the cultural traditions of society“ als die öffentliche Eisenbahn und Rolls-Royce, da seine europäische Geschichte zweieinhalbtausend Jahre bis hin zu jenen sagenhaften Griechen zurückreicht, die das Theater auch durch die Institution des Theorikons subventionierten, damit sich die ärmeren Bürger den Besuch leisten konnten. ${ }^{2}$

Der institutionelle Rahmen des Theaters, die Spielregeln, setzt sich aus einer Reihe von Mythen zusammen, die Geschichten über die Bedeutung kultureller Traditionen erzählen und damit einen Legitimitätsanspruch als Voraussetzung für - in den meisten Fällen - öffentliche Unterstützung erheben.

\section{Sechs Legitimationsmythen}

Legitimationsmythen sind reihenweise im Umlauf. Je nachdem ob sie ökonomisch oder moralisch ausrichtet sind, begründen sie Theater in Kategorien eines Gutes oder des Guten. Sie werden im Folgenden in einer Linie von „Güterabwägungen“ beschrieben, wobei der zugrunde gelegte Begriff des Guts stärker an ökonomische Theorien und weniger an philosophisch-ethische Begriffsbestimmungen angelehnt ist. Sie werden in chronologischer Reihenfolge behandelt.

\subsection{Theater als Moralisches Gut}

In der Wirtschaftstheorie gehören moralische Güter zu einer Kategorie von Gütern, die nicht marktfähig sind bzw. für die sich das Konkurrieren zwischen Marktteilnehmer*innen nicht lohnt. Bereits Adam Smith (2007) musste feststellen, dass die Arbeit von Schauspieler*innen, Sänger*innen oder Tänzer*innen über keinen Tausch- und Mehrwert - ,the work of all of them perishes in the very moment of its production“ (2007, S. 259) - aber dennoch über ,a certain value“ verfügt. Sie gehören, wenn sie wirtschaftstheoretisch überhaupt satisfaktionsfähig sind, zu den meritorischen und öffentlichen Gütern (vgl. Abschn. 4 unten). Da sie dennoch als wertvoll erachtet werden, muss eine externe Instanz, normalerweise

\footnotetext{
${ }^{2}$ Es handelt sich um eine Art Fonds, der finanzielle Hilfe für die ärmeren Stadtbürger bereitstellte, damit sie an den Aufführungen teilnehmen konnten. Der Althistoriker August Boekh bezeichnete die Theorikongelder als „Krebs der Athenischen Staatswohlfahrt“ (1851, S. 306).
} 
der Staat bzw. die öffentliche Hand sie bereitstellen. Diskutiert werden sie daher meistens unter werttheoretischen und ethischen Gesichtspunkten. Im Zusammenhang mit dem Theater als Institution hat der Begriff sowohl eine ökonomische als auch eine philosophisch-ethische Komponente; letztere wurde z. B. in der Unterscheidung zwischen dem „Guten“ und dem „Wahren“ verhandelt.

Debatten um seine Legitimität begleiten das Theater seit der Antike und dem Aufkommen des antitheatralischen Vorurteils (Barish 1981; Wild 2003). Von Platon bis zu den Puritaner*innen galt das Theater in einflussreichen, vor allem kirchlichen Kreisen als Ort der sittlichen Korrumpierung, nicht der Verbesserung. Im England des 17. Jahrhunderts führte diese Diskussion zur völligen Delegitimierung und der 1642 verfügten und bis 1660 andauernden Schließung aller Theater. Wie in England entstanden im 17. Jahrhundert auch in den deutschsprachigen Regionen und in Frankreich theaterfeindliche Strömungen, die in Schriften wie der Theatromania (1681) des pietistischen Pfarrers Anton Reiser oder den Maximes et réflexions sur la comédie (1694) des Franzosen Jean-Bénigne Bossuet ihren Niederschlag fanden. In der Schweiz verzichtete die Stadt Genf und eine Reihe anderer Kantone gänzlich auf Theaterhäuser und -truppen. Auch in deutschsprachigen Ländern - besonders in den stark pietistisch geprägten südwestlichen Regionen - blieben solche Argumente im 17. und 18. Jahrhundert virulent (Brandstetter et al. 2011). ${ }^{3}$

Für die Theaterreformer der Spätaufklärung bestand dann jedoch kein Zweifel mehr darüber, dass der Zweck der Schaubühne in ihrer sittlich-bildenden Wirkung zu sehen sei. Sie wurde als ,des sittlichen Bürgers Abendschule“ tituliert (HaidePregler 1980) und die Idee vor allem in den „,moralischen Wochenschriften“, dem Inbegriff einer bürgerlich-rationalen Öffentlichkeit, entwickelt und ausgefochten. Doch war der institutionelle Rahmen nicht unproblematisch: die einzigen Träger, die über zuverlässige Munifizenz verfügten, waren die Fürstenhöfe, während Wandertruppen, die keinen höfischen Auftrag ergattern konnten, von Stadt zu Stadt ziehen und mit den städtischen Behörden über Spielerlaubnisse verhandeln mussten, um ihr hybrides Repertoire (Haupt- und Staatsaktion, Stegreifkomödien, Tanz) präsentieren zu dürfen. Das Sesshaft-Werden der Theatertruppen, das Mitte des 18. Jahrhunderts einsetzt, geht mit diskursiven Entwicklungen einher, die die moralische Verbesserungsfunktion und den vermeintlichen Bildungsauftrag des Theaters zementiert. Während die meisten Beiträge die bekannten Argumente einfach wiederholen, ragen zwei Publikationen heraus, nicht nur, weil sie moralische

\footnotetext{
${ }^{3}$ Der deutsche Pietismus wirft auf das Theaterwesen immer noch einen langen Schatten. Zum Beispiel gibt es in ganz Baden-Württemberg keine einzige Professur für Theaterwissenschaft, geschweige denn ein Institut.
} 
Argumente mit institutionellen Überlegungen verknüpfen, sondern weil sie im 19. Jahrhundert den Status von Gründungsmythen erlangen: Lessings Hamburgische Dramaturgie (1769) und Schillers Aufsatz „Die Schaubühne als moralische Anstalt betrachtet" (1784).

Lessing ist für die vorliegende Fragestellung deshalb wichtig, weil seine „dramaturgischen“ Kommentare mit einem neuen Organisationsmodell, nämlich einer bürgerlichen Aktiengesellschaft in Zusammenhang stehen. Auch wenn die in der Hamburgischen Dramaturgie versammelten Beiträge finanzielle Fragen nur am Rande tangieren, bilden sie die diskursive Grundlage für das künftige Stadtheater unabhängig von fürstlich-höfischer Bevormundung. Bei der „Hamburgischen Entreprise“, handelte es sich, wie der Name schon sagt, um ein vom Bankrotteur, aber theateraffinen Spekulanten Abel Seyler finanziertes, zwischen Mäzenatentum und Gewinnabsicht oszillierendes Privatunternehmen. Obwohl die „Entreprise“ pleite ging, wanderte die mit ihr propagierte Idee eines von „welschen" Einflüssen gereinigten Nationaltheaters weiter. Hamburgische Dramaturgie und Nationaltheateridee sind untrennbar miteinander verknüpft, auch wenn Lessing den Begriff am Ende der Schrift in einem resignativen Duktus überhaupt erst verwendet, als schon klar ist, dass das Unternehmen (und für Lessing das Publikum) gescheitert ist. ${ }^{4}$ Die berühmte Sehnsuchtsklage - „Über den gutherzigen Einfall, den Deutschen ein Nationaltheater zu verschaffen, da wir Deutsche noch keine Nation sind! Ich rede nicht von der politischen Verfassung, sondern bloß von dem sittlichen Charakter" (Lessing 1981, S. 509, Hervorhbg. C.B.) - fand ein Jahrhundert lang Widerhall.

Auf wohlwollende Aufnahme traf sie aber zunächst in aufgeklärten höfischen Kreisen: erst in Wien, wo das Burgtheater 1776 in ein „Teutsches Nationaltheater", und ein Jahr später in Mannheim, wo das dortige Hoftheater in eine „Nationalschaubühne“ umbenannt wurden. Dort hielt Schiller im Rahmen seiner Tätigkeit als Theaterdichter seine berühmte, vor der kurpfälzischen Deutschen Gesellschaft gehaltene Rede, die sich mit der Leitfrage befasste: „Was kann eine gute stehende Schaubühne eigentlich bewirken?“ Der Begriff ,,stehend“ verweist auf die zunehmende Sesshaftigkeit der Truppen bzw. das Argument, dass erst durch das Sesshaft-Werden die (mit den umherziehenden Truppen assoziierten) moralisch-sittlichen Gefahren gebannt werden könnten. Allerdings bemüht Schiller sowohl ästhetische als auch moralische Argumente für institutionelle Zwecke:

\footnotetext{
${ }^{4}$ In seiner Abrechnung am Ende der „Hamburgischen Dramaturgie“ gibt Lessing neben einer unzulänglichen Schauspielkunst dem Publikum die Schuld am Scheitern des Unternehmens: ,und was hat denn das Publikum getan, damit etwas geschehen könnte? Auch nichts; ja noch schlimmer als nichts [...] es hat ihm [dem Unternehmen] nicht einmal seinen natürlichen Lauf gelassen“ (Lessing 1981, S. 509).
} 
er plädiert für staatliche Unterstützung, weil sich das Theater verbessernd auf den ganzen Staat auswirke: „Die Schaubühne ist der gemeinschaftliche Kanal, in welchen von dem denkenden bessern Teile des Volks das Licht der Weisheit herunterströmt und von da aus in milderen Strahlen durch den ganzen Staat sich verbreitet.“ (Schiller 1995, S. 10, Hervorhbg. C.B.).

Mit dem Hinweis auf "Gemeinschaft" leitet der Mythos der moralischen Anstalt über zur Nationaltheateridee. Im 19. Jahrhundert wandelt sich Schillers Neuformulierung alter moralischer Argumente in die Idee von Theater als Nukleus von Gemeinschaftsbildung.

\subsection{Theater als Gemeinschaft}

Im Zuge des aufkeimenden politischen und kulturellen Nationalismus wird das Theater zunehmend als Ort der Communitas vorgestellt: Es kann sogar als Synekdoche der Nation funktionieren, wie das Schiller-Zitat suggeriert: Von einer ideellen, konzentrierten Keimzelle verbreite sich das Gute und Wahre vom „,bessern Teile des Volks" auf den ganzen Staat. Das Theater wird als Bestandteil eines medialen Kommunikationsnetzes konzipiert, als Teil der ,imaginierten Gemeinschaft" in der Terminologie von Benedict Anderson (1983/1991), der argumentiert, dass Medien die Voraussetzung seien, dass Menschen, die sich nie kennenlernen werden, sich als eine Gemeinschaft der Nation fühlen. Der Mythos des Theaters als Ort der (nationalen) Gemeinschaft entfaltet im 19. Jahrhundert beachtliche Zugkraft und führt als Legitimationsmythos zur Gründung von Nationaltheatern überall in Europa, vor allem im heutigen Osteuropa, wo in den neuen, nach Unabhängigkeit strebenden „Volksgemeinschaften“ Nationaltheater errichtet wurden, teilweise bevor sie vollständige politische Unabhängigkeit erreicht hatten.

Ein Nationaltheater wird in der Regel als eine zweckbestimmte Struktur definiert, die die Nation repräsentieren soll und in der Regel durch staatliche Subventionen unterstützt wird. Metonymisch steht es für den Staat und die Nation in kulturellen Angelegenheiten. In Deutschland selbst, das erst 1871 eine vereinigte Nation bildet, fehlte diese metonymische, politische Funktion, weil es in deutschen Landen von Mannheim bis München überall Nationaltheater gab. Mit Ausnahme von Mannheim, wo bereits 1837 das Hoftheater in kommunale Trägerschaft überging, blieb diese Funktion den Hoftheatern überlassen, die ihre finanziellen Verpflichtungen über die Zivilliste, die Aufwendungen für den herrschaftlichen Haushalt, bestritten. Die ,nationale“ Verpflichtung konnte man an der Namensgebung ablesen, wie etwa das „Königliche Hof- und National-Theater“ in München. Am Vorabend der 1848er Revolutionen war das Nationaltheater 
jedoch nur noch Idee, ohne feste institutionelle Verankerung. Der Begriff bezog sich, einer Definition des Allgemeinen Theater-Lexikons (1841) zufolge, auf ,eine Bühne, die ausschließlich Stücke gibt, die ihrem Volke gehören“. Der Artikel musste aber resignativ feststellen: „Wir haben in Deutschland kein Institut dieser Art (vergleichbar dem Théâtre français oder Covent Garden), was (...) an dem Mangel der Nationalität selbst liegt (...). Ein Nationaltheater können wir erst dann erhalten, wenn es wieder ein Deutschland gibt.“ (Herloßsohn et al. 1841, S. 345).

Das Nationaltheater wurde aber auch als Organisationsstruktur in dieser Zeit neu gedacht: „Nur durch staatliches Einschreiten sei dem Theater zu helfen“, schloss der Schauspieler und spätere Theaterhistoriker Eduard Devrient (1874, S. 316) aus Schriften von „Bühnenverständigen“ der Restaurationszeit . Devrient selbst war 1848 während der Märzrevolution vom preußischen Kultusminister Adalbert von Ladenberg beauftragt worden, Reformideen zur Reorganisation der Theaterkunst zu formulieren. Das Ergebnis war eine kontrovers diskutierte Reformschrift mit dem programmatischen Titel „Das Nationaltheater des Neuen Deutschlands“ (Devrient 1849). Hier wurde im Sinne Hegels das Drama als „Kunst der Künste“ und als „Spitze der Pyramide“ definiert (Devrient 1849, S. 9). Devrient argumentierte, der große Idealismus der Nationaltheateridee wurde im Zuge der Restauration nach 1815 aufgegeben - der Name "Nationaltheater" sei überall durch die Titel „Hoftheater“ ersetzt - und die künstlerische Gestaltung der Theaterdirektoren durch kleinkrämerische, kunstfremde Hofintendanten als Aufsichtspersonen verdrängt: ,,das Bureau wurde nun der Mittelpunkt der Kunsttätigkeit" (1849, S. 15). Devrient argumentiert, dass ein echter, der Theaterkunst verpflichteter Intendant sich von rein finanziellen Erwägungen nicht beschränkt fühlen sollte: ,dass es nicht darauf ankommt: wie viel oder wie wenig ausgegeben, sondern was für das ausgegebene geleistet wird“ (1849, S. 20). Um diese finanzielle Freiheit zu gewährleisten, sollte ,bei der bevorstehenden Regulierung der Zivilliste (...) die Theatersubvention von derselben abgelöst und auf den Etat des Cultusministeriums übertragen werden, und sämtliche Bühnen des preußischen Staates dem selben Ministerium unterstellt" (Devrient 1874, S. 317-18). ${ }^{5}$

Entscheidend für die Bedeutung der Nationaltheateridee war jedoch weniger ihre Organisations- und Finanzstruktur als deren nationalistische, gemeinschaftsprägende Schlagkraft. Eine in Hamburg auf dem Höhepunkt der Schleswig-Holstein-Krise gehaltene Rede des an der 1848er Revolution in Wien beteiligten, österreichischen Dramatikers Ludwig Eckhardt steht pars pro toto für die ,politische Verfassung“ (Lessing) der Idee:

\footnotetext{
${ }^{5}$ Zum Kontext von Preußen als Kulturstaat vgl. Neugebauer (2009).
} 
„Ein deutsches Nationaltheater auf freiem deutschen Boden, ein von der ganzen Nation mitbegründetes, den Schwankungen des Tages enthobenes, idealen Richtungen ausschließend geweihtes Institut, das geistige Auge der Nation. Heil der Stadt, welche diese Krone, mit der unser Volk sich selbst einst noch schmücken soll, - vielleicht nach dem Siege der großen Sache - besitzen wird“ (Eckhardt 1864, S. 27).

Der „Sieg der großen Sache“ war die von Bismarck vorangetriebene Vereinigung Deutschlands, die 1864 zum Krieg mit Dänemark führte. Die Gründung des Deutschen Reichs 1871 brachte institutionsgeschichtlich gesehen kein Nationaltheater „auf freiem deutschen Boden“, sondern die Entfaltung der freien Marktwirtschaft. Das Preußische Gewerbeordnungsgesetz von 1869, das 1871 auf das gesamte neu gegründete Deutsche Reich ausgedehnt wurde, hob die Beschränkungen für die Gründung neuer Theater auf und schuf die Grundlage für einen marktwirtschaftlichen Wettbewerb (Gewerbefreiheit). Dies führte zu einem Boom im Theaterbau und einer Ausbreitung von Theaterhäusern, insbesondere in den größeren Städten wie Berlin. Nun wurden Theater und Unterhaltungsstätten aller Art als Gewerbe eingestuft und deren Genehmigung und Betrieb erheblich vereinfacht. Anstelle von ,geweihten Instituten“ entwickelte sich eine hochgradig ausdifferenzierte Theaterlandschaft, die von Tingeltangel bis zum Hoftheater ein großes Spektrum an darstellerischen Angeboten umfasste. Die bedeutendste institutionelle Neuerung war die Gründung von zahlreichen Stadttheatern als Aktiengesellschaften. 1914 gab es 196 Stadttheater - 118 davon im städtischen Besitz, 78 im Privateigentum. Die allermeisten wurden nach 1871 erbaut. Allein zwischen 1900 und 1913 entstanden 38 Stadttheater (Balme 2010, S. 64). Die Theater befanden sich im städtischen Besitz, die die Gebäude an privatwirtschaftlich arbeitende Theaterunternehmer verpachteten. Zählt man die 176 Privatbühnen hinzu, so wird deutlich, dass das deutsche Theatersystem vor dem Ersten Weltkrieg vor allem ein kommerzielles Unterfangen war, das allerdings zum Teil durch die Städte bezuschusst wurde.

Mit der Expansion ,legitimer Theater', d. h. Theater mit Erlaubnis, Theaterstücke aufzuführen, bildete sich im Laufe des 19. Jahrhunderts ein Organisationsfeld heraus, das die einzelnen Organisationen (Theater) noch stärker miteinander verknüpfte. Der neoinstitutionellen Theorie von DiMaggio und Powell (1983) zufolge entstehen bei einer Vielzahl von Akteuren mit ähnlichen Zielen Organisationsfelder, die eine Angleichung (Isomorphismus) der Akteure zur Folge haben: „In the initial stages of their life cycle, organizational fields display considerable diversity in approach and form. Once a field becomes well established, however, there is an inexorable push towards homogenization" (1983, S. 148). Isomorphismus manifestiert sich auf verschiedene Art und Weise, aber wohl am deutlichsten 
im Bereich der Professionalisierung, wo Berufsverbände gebildet, Ausbildungsinstitute gegründet und Führungskräfte ausgetauscht werden, um die Komplexität des Feldes zu bewältigen. Erste Anzeichen hin zum institutionellen Isomorphismus sind in der Gründung des Deutschen Bühnenvereins 1846 zu sehen, einem Zusammenschluss von Theaterdirektoren und Hoftheater-Intendanten, um u. a. dem „Unwesen“ der Theateragenten oder vertragsbrüchiger Schauspieler*innen zu begegnen - und dann 1871 die Etablierung einer Gewerkschaft, der Genossenschaft Deutscher Bühnen-Angehöriger, um den Arbeitnehmenden eine Stimme zu geben. Auch in diese Zeit fallen die ersten Versuche, staatliche Schauspielschulen ins Leben zu rufen (Ernst 2009). ${ }^{6}$ Auch wenn das Feld recht heterogen war - Hoftheater, Stadtheater im städtischen und Privatbesitz, reine Privattheater - funktionierte die Dynamik des Isomorphismus so gut, dass Bühnenschaffende einschließlich der Führungskräfte leicht zwischen den einzelnen Organisationen wechseln konnten.

Mit Ausnahme der 39 Hoftheater, deren Angestellte zum Teil verbeamtet und sogar pensionsberechtigt waren, waren die Arbeitsbedingungen oft katastrophal. Am Vorabend des Ersten Weltkriegs sprach man von einem „Bühnenproletariat“ (Engel-Reimers 1911, S. 318), und vom Legitimierungsmythos der nationalen Gemeinschaft war wenig übrig. ${ }^{7}$ Er wurde ab 1900 von einem neuen Mythos abgelöst.

\subsection{Theater als öffentliches Gut}

Das Volk wird nun zur Öffentlichkeit. Dies ist der vorherrschende theatrale Legitimierungsmythos des 20. Jahrhunderts. Er rechtfertigt die Verwendung von Subventionen, d. h. Steuergeldern zur Finanzierung des Theaters oder bestimmter Theaterarten: Die Subventionsdebatte stützte sich auf sozialdemokratische Vorstellungen, wonach Theater als integraler Bestandteil des Wohlfahrts- bzw. Sozialstaates zu betrachten sei; d. h. die Bereitstellung von Theater sei gleichwertig mit anderen öffentlichen Einrichtungen.

\footnotetext{
${ }^{6}$ Versuche, Schauspielausbildung zu verstaatlichen, im tertiären Bereich anzusiedeln, wären nach DiMaggio und Powell klare Anzeichen für normativen Isomorphismus: ,the resting of formal education and of legitimation in a cognitive base produced by university specialists" (1983, S. 152).

${ }^{7}$ Eine entpolitisierte Version der theatralen Gemeinschaft wurde in den letzten Jahren vornehmlich von deutschen Wissenschaftler*innen neu formuliert, und zwar in rein ästhetischen Begriffen einer vergänglichen, transitorischen Gemeinschaft von Gläubigen auf dem Altar des Hier und Jetzt, die an der Ästhetik der Präsenz teilhaben.
} 
Der Ruf nach staatlicher Unterstützung beginnt bereits, wie wir gesehen haben, im Zeichen der 1848er Revolution, wobei zwischen höfischer (über die Zivilliste) und staatlicher (Steuergelder) Finanzierung unterschieden wird. Erst gegen Ende des 19. Jahrhunderts erweitert sich der Diskurs, um auch die neuen Stadttheater einzubeziehen. Hintergrund war ein mit erheblicher Vehemenz geführter publizistischer Kampf um die Alternative ,Geschäftstheater' versus ,Kulturtheater'. Für die Verfechter der Kulturtheateridee verkörperte das Geschäftstheater alle Übel, die das damalige Theaterwesen auszeichneten: von anspruchslosen Spielplänen über Virtuosentum bis hin zu schlechten Arbeitsbedingungen für die Bühnenangehörigen. Die seit einem Jahrhundert intellektuell vorbereitete Erhebung des Theaters in den Ritterstand der Künste, seine Verklärung zum Kunsttempel, drohte immer wieder an der allabendlichen wirtschaftlichen Realität der Theateraufführungen zu scheitern (Leonhardt 2007, S. 122 f.).

Die zentralen Argumente der Kulturtheaterfraktion finden sich in einer von Ludwig Seelig verfassten Denkschrift mit dem Titel Geschäftstheater oder Kulturtheater? (Seelig 1914). Als Syndikus des Kartells der Verbände der deutschösterreichischen Bühnen- und Orchestermitglieder machte der Sozialdemokrat Seelig keinen Hehl aus seiner Vorstellung für die Zukunft der Stadt- und Staatstheater. 1918 schrieb er im sozialdemokratischen Parteiorgan Vorwärts: „Es gibt nur ein Mittel, um das Theater seiner Kulturbestimmung zuzuführen: das ist seine Sozialisierung!“ (Seelig zitiert nach Bullinger 1997, S. 141). Der Ruf nach einer Sozialisierung des Theaters fand in der Weimarer Republik großen Widerhall, zumal das alte Modell der verpachteten Stadtheater an seine gewinnbringenden Grenzen stieß. Einen verheißungsvollen Beginn machte die Münchner Räterepublik. Der vom Schauspieler Albert Florath am 3. Januar 1919 auf der neunten Sitzung des Provisorischen Nationalrats eingebrachte Antrag zur ,Besserung der Lage aller künstlerischen Berufe" enthielt zahlreiche radikale Forderungen, die unter dem Begriff „Sozialisierung des Theaters“ zusammengefasst wurden. Florath erklärte schon am Anfang seiner langen Rede: „für den Kapitalismus ist das Theater ein Vergnügungsmittel, für den Sozialismus ist das Theater eine Kulturanstalt, die Theaterkunst eine Kulturnotwendigkeit." (Verhandlungen des Provisorischen Nationalrates des Volksstaates Bayern 1919, S. 266) Konkret bedeutete Sozialisierung die Verstaatlichung aller (Kultur-)Theater, Abschaffung der Theateragenten, Gründung einer staatlichen Hochschule für Schauspielkunst, Schaffung einer staatlichen Kranken-, Unfall-, Invaliden-, Alters- und Arbeitslosenversicherung für Künstler*innen, Festlegung einer Minimalgage. Obwohl der mit großer Mehrheit verabschiedete Antrag ungefähr die gleiche Nachhaltigkeit hatte wie die Räterepublik selbst, wurden über die Jahre alle Forderungen letztlich umgesetzt. So wurden bereits 1927 die privaten Theateragenten abgeschafft 
und eine staatliche Einrichtung, die Reichsanstalt für Arbeitsvermittlung, der VorVorläufer der heutigen Zentralen Arbeitsvermittlung für Künstler (ZAV-Künstler) gegründet, um Theatervermittlung den Gesetzen des Marktes zu entziehen. Eine vollständige Kommunalisierung aller Stadtheater wurde im Laufe der 1930er Jahre unter NS-Herrschaft erreicht (Balme 2010, S. 75).

Der Legitimierungsmythos des Theaters als öffentliches Gut blieb nicht auf Deutschland beschränkt, obwohl seine globale Verbreitung und Institutionalisierung erst nach dem Zweiten Weltkrieg erfolgten. Die Grundgedanken hinter dem Mythos wurden 1954 von Jean Vilar, Regisseur und Gründer des Théâtre National Populaire in Frankreich, prägnant formuliert: „Das Théâtre National Populaire ist in erster Linie eine öffentliche Einrichtung, genau wie Gas, Wasser, Strom“ (Vilar 1975, S. 173). Vilar stellte sich das Theater als ein öffentliches Gut vor, das, frei von den Launen des Marktes, den gleichen Status wie Grundbedürfnisse und öffentliche Dienstleistungen haben sollte. Der Unterhalt von Theatern und Orchestern gehörte neben der Bereitstellung von Gas, Wasser und Strom zur „Daseinsvorsorge“. 8 Damit konnten zumindest in Deutschland, aber auch in Frankreich große Teile des Theaters aus dem kulturindustriellen System herausgelöst und als Aufgabe der öffentlichen Grundversorgung umdefiniert werden. Die Kommunalisierung des Theaters hatte logischerweise zur Folge, dass es weitgehend lokal wahrgenommen wurde.

Nach 1945 vollzieht sich selbst in den USA in der Kulturförderung eine bedeutende Verschiebung von Profit zu Non-Profit (DiMaggio 1992; Toepler und Zimmer 2002). Bis Ende der 1960er Jahre hatte der größte Teil Westeuropas und sicherlich alle osteuropäischen und anderen sozialistischen Länder Theaterinstitutionen einschließlich Ausbildungsstätten öffentlich subventioniert. Dies ist in den meisten europäischen Ländern nach wie vor der vorherrschende Mythos, aber besonders im Vereinigten Königreich bildet er eine problematische Koexistenz mit einem anderen Legitimierungsmythos, nämlich jenem, dass das Theater und die Kultur insgesamt einen bedeutenden ökonomischen Beitrag zum Bruttosozialprodukt leisten.

\subsection{Marktgüter}

Das Theater als dynamischer Marktteilnehmer fußt auf der Theorie der Umwegrentabilität, einem Begriff aus der Ökonomie, der - wie ein Zoonose-Virus - in

${ }^{8}$ Der Begriff der „Daseinsvorsorge“ als Aufgabe der öffentlichen Verwaltung wurde vom Verwaltungsrechtler Ernst Forsthoff während der NS-Zeit eingeführt (Meinel 2007). 
den 1970er Jahren in den kulturellen Bereich überspringt und im Zeichen neoliberalen Denkens in den 1990er Jahren große Verbreitung findet. ${ }^{9}$ Diesem Mythos zufolge ist das Theater nicht nur Empfänger öffentlicher Unterstützung (gleichsam ein im Endstadium der Baumol'schen Kostenkrankheit leidender Patient), sondern durch die damit verbundenen Neben- und Folgeeffekte (Gastronomie, Hotels, Merchandising) ein dynamischer Generator wirtschaftlicher Aktivität und ein Pull-Faktor für gut qualifizierte Arbeitskräfte. Der Mythos der Umwegrentabilität liefert eine wichtige Rechtfertigung für Festivals, die sich in den 1990er Jahren als eine Art Allheilmittel für den regionalen Niedergang ausbreiteten, als Städte und Dörfer verzweifelt nach Belegen dafür suchten, dass ein berühmter Schriftsteller, Komponist oder Künstler dort eine gewisse Zeitspanne verbracht hat (um somit eine Rechtfertigung für Händel-, Bach-, Shakespeare-Festivals zu liefern). Wir finden das Argument jedoch bereits 1919 bei den von Max Reinhardt und Hugo von Hofmannsthal ins Leben gerufenen Salzburger Festspielen. In seinem Antrag an den Generalintendanten des kaiserlichen Hoftheaters Wien betonte Max Reinhardt nicht nur die künstlerischen, sondern auch die wirtschaftlichen Vorteile. Ein solches Festival, so argumentierte er, sei ein Aushängeschild für wohlhabende Touristen aus dem In- und Ausland, die einen wesentlichen Beitrag zur Wirtschaft der Region leisten würden (Reinhardt 1974).

Die meisten Festivals erhalten auch öffentliche Gelder; sie werden als ein Mittel angesehen, um Touristen aus dem In- und Ausland anzuziehen, und daher werden die öffentlichen Gelder als Investition betrachtet. Mit diesem Argument wird der Legitimierungsmythos der öffentlichen Güter weitgehend in den Mythos der Marktgüter absorbiert. Er wird als Basis- oder Anschubfinanzierung gerechtfertigt, die im Sinne von Startkapital langfristig eine Mehrfachrendite abwerfen soll. Diese Argumentationsfigur bleibt jedoch nicht auf das Festivalwesen beschränkt, sondern findet begrenzt auch im öffentlichen Bereich Aufnahme. Am radikalsten wurde der Wandel vom öffentlichen zum marktfähigen Gut wohl in Großbritannien vollzogen, wo der Anteil an staatlicher Förderung seit Thatcher immer weiter zurückging (Kershaw 1999), bis sie heute beim National Theatre beispielsweise nur noch ca. $25 \%$ ausmacht.

Auch in Deutschland hat sich diese Argumentationsfigur etabliert, und zwar seit Mitte der 1990er Jahre, allerdings ohne ernsthaft den institutionellen Rahmen und Legitimierungsmythos des öffentlichen Guts infrage zu stellen. Am nachhaltigsten etabliert hat er sich in den Studiengängen für Kulturmanagement, in

\footnotetext{
${ }^{9}$ Wie bei Corona ist der Ursprung des Umwegrentabilitäts-Virus umstritten. In Deutschland hat wahrscheinlich ein Gutachten des Münchner Ifo-Instituts (Hummel und Berger 1988) eine entscheidende Rolle gespielt. Vgl. hierzu Heinrichs (1997, S. 33).
} 
denen seit den 1990er Jahren Ansätze zum betriebswirtschaftlichen Denken in die kritische Analyse des etablierten Systems der öffentlichen Theaterfinanzierung Eingang gefunden haben. Obwohl nicht alle Vertreter*innen des Fachs einer rein managerialen Denkweise das Wort reden, wurde die Grundeinstellung in der bewusst polemisch formulierten und von vier Fachvertretern verfassten Publikation, Der Kulturinfarkt: Von Allem zu viel und überall das Gleiche (Haselbach et al. 2012) zum Ausdruck gebracht. Sie fordern die Hälfte aller Kulturinstitutionen $\mathrm{zu}$ schließen, und die daraus freiwerdenden finanziellen Mittel in die Erweiterung des künstlerischen Angebots jenseits der etablierten Kulturorganisationen wie Museen, Theater und Konzerthäuser zu ,investieren“. Obwohl das Buch vom Feuilleton und von Wortführenden des Kulturbetriebs schnell und gnadenlos zerrissen wurde, kam es, zumindest für eine kurze Zeit, zu einer öffentlichen Diskussion über die Legitimität des etablierten Kulturbetriebs. Jenseits der Polemik auf beiden Seiten erwies eine genaue Lektüre des Buchs, dass Forschungsdiskussionen vor allem aus der Disziplin der Kulturökonomie bzw. einer nachfrageorientierten Perspektive verarbeitet wurden. So findet man Argumente, dass meritorische bzw. öffentliche Güter wie ,normale“ Wirtschaftsgüter, die zwischen Angebot und Nachfrage ein Äquilibrium finden, behandelt werden sollten. Bereits die Enquete-Kommission des Deutschen Bundestags hat festgestellt, dass ,die Angebotsorientierung ein Kennzeichen des Kulturbereichs und der Kulturpolitik“" ist und nicht die Nachfrage (Dt. Bundestag 2007, S. 222). Zusammenfassend lässt sich feststellen, dass der Legitimationsmythos des marktfähigen Guts auf ganz ausgewählte Bereiche des Kulturlebens wie etwa Festivals und in einem bestimmten akademischen Diskurs beschränkt blieben. Ein neuerer Legitimationsmythos hatte sich parallel etabliert, nämlich Theater als soziales Gut.

\subsection{Theater als soziales Gut}

Ende des 20. Jahrhunderts entsteht eine aktualisierte Version des Mythos der moralischen Anstalt. Wenn nicht das Theater selbst, dann könnten dessen Kerntätigkeiten, die die künstlerische Produktion ausmachen - Schauspielkunst, Rollenspiel, kooperative Produktionsformen und vor allem das interaktive Spiel mit Zuschauern - für soziale, wenn nicht sogar therapeutische Zwecke genutzt werden. Dieses instrumentelle Verständnis von Theater ist das Herzstück der meisten Formen des angewandten Theaters, aber wir finden es in Variationen in allen Formen der Vermittlung, in Bildungsprogrammen, in der Theaterpädagogik (Pinkert und Sack 2014; Primavesi und Deck 2014; Kup 2019). Es gibt Stimmen 
(Bishop 2004), dass solche sozialen Ansprüche vielleicht zur Delegitimierung des Theaters und der Kunst beitragen, zumindest in seinen traditionellen Formen der Unterhaltung in abgedunkelten Zuschauerräumen vor zahlendem Publikum. Dieser Legitimationsmythos hat zur Bildung des autonomen organisatorischen Bereichs des angewandten Theaters (,,applied theatre") geführt, mit verschiedenen, inzwischen hoch spezialisierten Unterbereichen wie Theater for development (TfD) oder das von Augusto Boal initiierte „Theater der Unterdrückten“ (TO) (Andrade und Balme 2020).

Demgegenüber sehr der Differenzierung und Aktualisierung bedürftig ist in Deutschland der lange sehr einflussreiche, mit dem Namen Hilmar Hoffmann verbundene Diskurs über „Kultur für alle“ (Benzer 2016), der hier nicht als eigener Legitimationsmythos behandelt wird (weil er recht pauschal auf dem Mythos des öffentlichen Gutes bezogen auf alle Kulturbereiche aufbaut).

Eine programmatische Grundlage dieses Mythos bildet die ungemein einflussreiche Schrift des französischen Kunstkritikers und Kurators Nicolas Bourriaud (1998) zur relationalen Ästhetik. Bei der Betrachtung zahlreicher künstlerischer Experimente, die Ereignisse und keine objekthaften Werke hervorbrachten, argumentierte er, dass bei solchen künstlerisch-relationalen Experimenten „Intersubjektivität“ das "Substrat“ des Kunstereignisses sei. Anstelle von Farbe, Leinwand oder Stein bilden die Beziehungen der Besucher*innen zum Werk oder untereinander die Grundlage der Kunsterfahrung. Bourriaud beschrieb eine existierende Kunstpraxis, die stark performative Elemente enthält und vor allem eine (inter-)aktivierende Rolle der Betrachtenden/Zuschauer*innen voraussetzte. Dies fügte sich auch sehr gut in neue kulturpolitische Strategien, die ebenfalls eine stärkere Publikumsorientierung bis hin zu Interventionen verlangte. Die englische Kunstkritikerin Claire Bishop warnt jedoch vor einem solchen social turn. ${ }^{10}$ Kunstpraktiken, die versuchen, soziale Übel zu korrigieren $-\mathrm{d}$. h. solche, die „Gutes tun“ - bergen die Gefahr einer übermäßigen Instrumentalisierung, die die formalästhetische Komplexität banalisiere und die interrogativen Möglichkeiten der Kunst unter dem homogenisierenden Schutzraum eines hehren sozialen Ziels aufhebe (Bishop 2004). ${ }^{11}$

Der Mythos manifestiert sich kulturpolitisch und institutionell am deutlichsten im Ausbau theaterpädagogischer Angebote an den Theatern, vor allem in den Bereichen des klassischen Konzerts und der Oper im Besonderen wie des

\footnotetext{
${ }^{10}$ Der Begriff „,social turn“ wird vor allem auf Entwicklungen in der Kunst bezogen, die soziale Beziehungen und weniger einzelne Werke thematisieren. Hierzu grundlegend Claire Bishop (2012).

${ }^{11}$ Vgl. auch Jackson (2011, S. 46-47).
} 
Musiktheaters im Allgemeinen (Tröndle und Behrens 2018; Plank-Baldauf 2019). Hintergrund ist hier zum einen der Wunsch, die recht hohe steuerfinanzierte Förderung dieser Einrichtungen möglichst vielen Bürger*innen zugutekommen zu lassen, und zum anderen dem noch viel dringenderen Problem eines ,enkulturativen" Bruchs im Stammpublikum zu begegnen. Ein enkulturativer Bruch bedeutet, dass ein deutlich zu beobachtender Publikumsschwund auf fehlende bzw. gestörte Prozesse bei der Aneignung und Weitervermittlung (hoch-)kultureller Praktiken zurückzuführen ist. Auch der demografische Wandel wird kulturpolitisch registriert (Dt. Bundestag 2007, S. 219-228), und die Frage gestellt, wie Theater darauf zu reagieren gedenken. Die Antwort liegt in der ,Vermittlung“, d. h. in der Schaffung neuer Berufsgruppen und Arbeitsbereiche, die sich institutionell als Figuren des Dritten (Korschorke 2010; Stauss 2020) von triadischen Beziehungen ausgehend manifestieren (in Abgrenzung zur herkömmlichen dyadischen Publikum-Künstler*innen-Beziehung). Vermittlungsstrategien tragen zur Legitimation von öffentlich getragenem Musiktheater bei, nicht nur indem sie hermeneutische Abhilfe leisten, sondern auch um in die ganze, mit einem Theaterund Konzertbesuch verbundene Sozialisation einzuführen. Das Theater als soziales Gut umfasst sowohl dezidierte interventionistische Maßnahmen wie ,applied theatre" als auch die Vermittlung von Theater in einem soziokulturellen Kontext.

Das Thema der Kultur- bzw. Theatervermittlung ist insofern stark zukunftsgerichtet, als die Kulturpolitik und damit der Hauptgeldgeber des Theaterwesens im deutschsprachigen Raum „Vermittlung“ als Lösung für die meisten anstehenden Legitimationsprobleme für kulturelle Institutionen entdeckt hat und unermüdlich propagiert. Vermittlung soll dafür sorgen, ein „Publikum der Zukunft“ zu gewinnen. Neuere Forschung (Prinz-Kiesbüye und Schmidt 2010) zeigt, dass die Propagierung der Vermittlung in ihren inzwischen recht komplex gewordenen Ebenen eine Krise der Kunstinstitutionen bloßlegen kann. Die neue Diskussion über Vermittlung legt nahe, dass die Institutionen selbst - die eigentlichen Vermittlungsinstanzen von Kunst - nun der Vermittlung bedürfen: wir befinden uns gleichsam auf einer Ebene der Vermittlung der Vermittlung.

\subsection{Theater als kreatives Gut}

Dieser Mythos ist mit progressivem, unabhängigem Theater verbunden oder ist manchmal gleichbedeutend mit dem Mythos der „Freien Szene“ (Matzke 2013). Seinem gemeinsamen Gründungsmythos liegt eine anti-institutionelle Haltung 
zugrunde, die sich im Engagement für kollektive Organisations- und Produktionsformen zeigt. Diese Akteure erlangen Legitimität durch den Anspruch, frei von institutionellen Zwängen, Produktions- und Probenplänen usw. zu sein.

Das „kreative“ Modell positioniert sich in Opposition zu autoritären Strukturen, favorisiert oft kollektiv erarbeitete Produktionen, stellt häufig die Figur des Regisseurs/der Regisseurin infrage (als Verkörperung und Perpetuierung autoritärer Strukturen) und lehnt die ,industrielle“ Produktion und Wiederholung von Inszenierungen ab. Die ästhetischen Formen und Formate sind vielfältig, aber das verbindende Element ist die Ablehnung der wahrgenommenen Kontrolle durch die „Tradition“, vor allem des inszenierten Stücks und des „Apparats“ des Staatsund Stadtheaters.

Die in der Freien Szene tätigen Künstler*innen stehen in einem manchmal spannungsgeladen Verhältnis zu Diskursen um Kreativität und Innovation, die beide stark von ökonomischen Logiken ausgehen und inzwischen unter dem Begriff „Kreativitätsdispositiv“ zusammengefasst sind (Reckwitz 2012). Trotz all ihrer Kreativität und Autonomie sind freie Gruppen von verschiedenen Finanzierungsregimes und damit institutionellen Regeln abhängig und existieren unter diesen. Alexander Pinto betrachtet die Freie Szene als „ein unter anderem durch eigene Spielstätten, Förderstrukturen, Medien, Veranstaltungen und Organisationen institutionalisiertes System innerhalb der darstellenden Künste" (Pinto 2013, S. 243). In Großbritannien und den Niederlanden zum Beispiel, wo die Regeln der Projektfinanzierung maßgeblich sind, machen solche Gruppen einen großen Teil der Förderungsempfänger*innen aus. In Deutschland hingegen bekommen sie relativ gesehen, d. h. im Vergleich zu den Staats- und Stadttheatern, kaum mehr als die Krümel vom Tisch. Einige dieser Krümel können ein ziemlicher Brocken sein, vor allem für die bereits etablierten freien Gruppen. Selbst bei einer ausgeprägten anti-institutionellen Haltung unterliegen unabhängige Gruppen also immer noch den Finanzierungsregimes und ihren Regeln und passen sich entsprechend an.

Die freien Theatergruppen positionieren sich selber, wie es opportun ist, auch im Zeichen kulturpolitischer Diskussionen um die „kreative Stadt“, die seit 2000 im Zuge der Erfindung der Kulturwirtschaft (Pinto 2013) einen neuen Diskurs mit Legitimationspotential markiert. Dies gilt wahrscheinlich zurzeit primär für Berlin, aber es gibt bereits Anzeichen, dass andere Städte wie Hamburg und Köln ihre „freien Kreativen“ ebenfalls als Wirtschaftsfaktor und nicht nur als Belastung betrachten.

In unmittelbarer Zukunft wird eine wachsende Verflechtung zwischen dem Mythos des öffentlichen Guts, verkörpert in den Staats- und Stadttheatern, und des kreativen Guts, repräsentiert durch die Freien Szene, von zentraler Bedeutung sein. Nicht nur weil es exogene kulturpolitische Maßnahmen wie das von 
der Bundeskulturstiftung aufgelegte Förderprogramm „Doppelpass“ gibt, sondern weil die Staats- und Stadttheater selber nach Wegen suchen, mit den Kreativen der Freien Szene zu kooperieren.

\section{$4 \quad$ Fazit}

Meyer und Rowan (1977) argumentieren, dass institutionelle Regeln als Mythen funktionieren oder die Mythen funktionieren als Regeln, die Organisationen einbauen, um Legitimität und damit Ressourcen, Stabilität und verbesserte Überlebensaussichten zu gewinnen. Wir haben gesehen, dass die Mythen, die institutionellen Regeln zugrunde liegen, nicht unveränderlich sind; und jede Verschiebung führt zu einer institutionellen Transformation. Ob wir eine solche Transformation als Krise betrachten, hängt in hohem Maße von der Perspektive der Akteure ab. Die organisatorischen Bereiche des Theaters haben sich zwischen 1900 und 1960 dramatisch verändert, als es von einer kapitalistischen Kulturindustrie in einen öffentlich finanzierten Teil der kulturellen Infrastruktur der meisten Länder umgewandelt wurde. Die industriell-kommerzielle Version hat nur in Megastädten wie New York, London und in Deutschland im Musical überlebt.

Die vielleicht weitestreichende Implikation der neoinstitutionalistischen Theorie besagt, dass die Bedeutung der formalen Struktur innerhalb von Organisationen nicht unbedingt mit einer besseren oder effizienteren Produktion korreliert. Die Befolgung der Regeln der formalen Struktur innerhalb von Organisationen führt nicht notwendigerweise zu besseren Ergebnissen. Hier kommen die Legitimitätsmythen als eine vernachlässigte Quelle der Funktionsweise von Organisationen ins Spiel: Letztere wenden beträchtliche Energie auf, um den Anforderungen der Mythen zu entsprechen, die durch organisationsexterne Akteure unterstützt werden (die die Mythen ebenfalls internalisiert haben):

\footnotetext{
„Many of the positions, policies, programs, and procedures of modern organisations are enforced by public opinion, by the views of important constituents, by knowledge legitimated through the educational system, by social prestige, by the laws (...)" (Meyer and Rowan 1977, S. 343).
}

Die hier aufgezählte Liste ist bezeichnend: Die öffentliche Meinung, wichtige Akteure (siehe Lobbyismus und ,pressure groups"), das Bildungssystem und sogar Gesetze spielen alle eine Rolle bei der Umsetzung der ,stark rationalisierten Mythen" in die Praxis. 
Das bestimmende Merkmal der institutionellen Mythen ist, dass sie in hohem Maße rationalisiert sind und daher unumstößlich erscheinen. Organisationen, insbesondere komplexe, unternehmen außergewöhnliche Anstrengungen, um Konformität mit den Regeln zu zeigen, was Meyer und Rowan „Zeremonie“ nennen, während gleichzeitig die Menschen vor Ort in den Organisationen diese Regeln oft untergraben oder umgehen, um die Organisation am Laufen zu halten.

Im Juni 2020 ist das Theater in mehrere, zum Teil widersprüchliche Mythen verstrickt: Theater als öffentliches Gut steht in einem potenziell konfliktträchtigen Verhältnis zu seiner Rolle als Marktgut. Dies ist in der Corona-Krise eklatant deutlich geworden. Besonders in Großbritannien ist das scheinbar akzeptable Gleichgewicht zwischen niedrigen Subventionen und hohen Einnahmen zusammengebrochen, sodass die meisten Theater vor der Schließung stehen, vielleicht für immer (trotz im Sommer 2020 von Regierungsseite in Milliardenhöhe zugesagten, aber vielerorts wohl zu spät kommenden Finanzhilfen). Der Ort des neuesten Mythos, des kreativen Gutes, ist immer noch ungeklärt, da seine „Effizienz" in Relation zu den großen staatlich bezuschussten Theatern aus finanzieller Sicht unbestritten ist. Die „Effizienz“ der neuen Kreativen kann als Mittel zur Delegitimierung der teuren Staats- und Stadttheater benutzt werden. Welche Kombinationen sich in den nächsten Jahren auch immer herausbilden mögen, wenn die Corona-Pandemie erst einmal bewältigt oder unter Kontrolle ist: Klar ist, dass die verbleibenden Organisationen weiterhin nach den Regeln spielen müssen, wenn auch nach modifizierten. Sie müssen sich auch genau darüber im Klaren sein, welche Legitimitätsmythen wie umgeschrieben werden.

\section{Literatur}

Anderson, Benedict. 1991. Imagined communities: Reflections on the origin and spread of nationalism. Revised. London: Verso.

Andrade, C. und C. Balme. 2020. Transnational networks of the theatre of the Oppressed: The institutionalization of a circulating method. Journal of Global Theatre History, 4 (1)3-20.

Balme, Christopher. 2010. Stadt-Theater: Eine deutsche Heterotopie zwischen Provinz und Metropole. In Großstadt. Motor der Künste in der Moderne, Hrsg. Burcu Dogramaci, 61-76. Berlin: Gebr. Mann.

Balme, Christopher. 2017. Repertoire and genre. In A cultural history of theatre, Hrsg. Balme, C. und T.C. Davis, Bd. 5, 181-201. London: Bloomsbury.

Barish, Jonas, und A. . 1981. The antitheatrical prejudice. Berkeley: University of California Press.

Benzer, Sabine, Hrsg. 2016. Kultur für alle: Gespräche über Verteilungsgerechtigkeit und Demokratie in Kunst und Kultur heute. Wien und Bozen: Folio Verlag.

Bishop, Claire. 2004. Antagonism and Relational Aesthetics. October 110 (Fall), 51-79. 
Bishop, Claire. 2012. Artificial Hells. Participatory art and the Politics of Spectatorship. London: Verso.

Boekh, August. 1851. Die Staatshaltung der Athener. Berlin: Reimer.

Bourriaud, Nicolas. 1998. Relational Aesthetics. Dijon: Les Presses du Réel.

Brandstetter, G., S. Diekmann, und C. Wild, Hrsg. 2011. Theaterfeindlichkeit. Anmerkungen zu einem unterschätzten Phänomen. München: Fink.

Bullinger, Matthias. 1997. Kunstförderung zwischen Monarchie und Republik: Entwicklungen der Kunstförderung in Württemberg zwischen 1900 und 1933 am Beispiel der Theater in Stuttgart, Ulm und Heilbronn. Frankfurt: Neue Wissenschaft.

Colyvas, J., und W.W. Powell. 2006. Roads to institutionalization: The remaking of boundaries between public and private science. Research in Organizational Behavior 27: 305-353.

Deutscher Bundestag. 2007. Schlussbericht der Enquete-Kommission „Kultur in Deutschland“. Deutscher Bundestag. https://dipbt.bundestag.de/dip21/btd/16/070/1607000.pdf. Zugegriffen: 15. Juni 2020.

Devrient, Eduard. 1849. Das Nationaltheater des neuen Deutschlands: eine Reformschrift. Leipzig: J. J. Weber.

Devrient, Eduard. 1874. Dramaturgische Schriften: Geschichte der deutschen Schauspielkunst: Das Virtuosentum. Leipzig: J. J. Weber.

DiMaggio, P.J., und W.W. Powell. 1983. The iron cage revisited: Institutional isomorphism and collective rationality in organizational fields. American Sociological Review 48 (2): 147-160.

DiMaggio, P.J., und W.W. Powell, Hrsg. 1991. The new institutionalism in organizational analysis. Chicago: University of Chicago Press.

DiMaggio, Paul. 1992. Cultural Boundaries and Structural Change: The Extension of the High Culture Model to Theater, Opera, and the Dance, 1900-1940. In Cultivating Differences: Symbolic Boundaries and the Making of Inequality, Hrsg. M. Lamont und M. Fournier, 21-57. Chicago: University of Chicago Press.

Eckhardt, Ludwig. 1864. Lessing und das erste deutsche Nationaltheater in Hamburg: Eine deutsche Gabe zur Shakespeare-Feier. Hamburg: Boyes \& Geisler.

Engel-Reimer, Charlotte. 1911. Die deutschen Bühnen und ihre Angehörigen: Eine Untersuchung über ihre wirtschaftliche Lage. Leipzig: Duncker \& Humblot.

Ernst, Wolf. D. 2009. „,Vorschrift und Affekt: Eine Diskursgeschichte der Schauspielausbildung zwischen 1870 und 1930" Habil-Schrift. München: LMU.

Haide-Pregler, Hilde. 1980. Des sittlichen Bürgers Abendschule: Bildungsanspruch und Bildungsauftrag des Berufstheaters im 18. Jahrhundert. Wien: Jugend und Volk.

Haselbach, D., A. Klein, P. Knüsel, und S. Opitz. 2012. Der Kulturinfarkt: Von allem zu viel und überall das Gleiche. München: Albrecht Knaus.

Heinrichs, Werner. 1997. Kulturpolitik und Kulturfinanzierung: Strategien und Modelle für eine politische Neuorientierung der Kulturfinanzierung. München: C.H. Beck.

Herloßsohn, C., H. Marggraff, und R. Blum, Hrsg. 1841. Allgemeines Theater-Lexikon: oder Encyklopädie alles Wissenwerthen für Bühnenkünstler, Dilettanten und Theaterfreunde., Bd. 5. Altenburg und Leipzig: Expedition des Theater-Lexikons.

Hummel, M. und M. Berger. 1988. Die volkswirtschaftliche Bedeutung von Kunst und Kultur. Gutachten im Auftrag des Bundesministers des Innern. In Schriftenreihe des Ifo-Instituts für Wirtschaftsforschung. Berlin: Dunckler \& Humblot. 
Jackson, Shannon. 2011. Social works: performing art, supporting publics. New York: Routledge.

Kup, Johannes. 2019. Das Theater der Teilhabe: zum Diskurs um Partizipation in der zeitgenössischen Theaterpädagogik. Berlin: Schibri.

Kershaw, Baz. 1999. Discouraging Democracy: British Theatres and Economics, 1979-1999. Theatre Journal 51 (3): 267-283.

Korschorke, Albrecht. 2010. Ein neues Paradigma der Kulturwissenschaften. In Die Figur des Dritten: ein kulturwissenschaftliches Paradigma, Hrsg. E. Eßlinger, T. Schlechtriemen, D. Schweitzer, und A. Zons, 9-31. Frankfurt a. M.: Suhrkamp.

Leonhardt, Nic. 2007. Piktoral-Dramaturgie: Visuelle Kultur und Theater im 19. Jahrhundert (1869-1899). Bielefeld: transcript.

Lessing, G.E. 1981. Hamburgische Dramaturgie. Stuttgart: Reclam.

Matzke, Annemarie. 2013. Das „Freie Theater“ gibt es nicht. Formen des Produzierens im gegenwärtigen Theater. In Theater entwickeln und planen. Kulturpolitische Konzeptionen zur Reform der Darstellenden Künste, Hrsg. W. Schneider, 259-272. Bielefeld: transcript.

Meinel, Florian. 2007. Der Jurist in der industriellen Gesellschaft: Ernst Forsthoff und seine Zeit. Berlin: Akademie.

Meyer, J.W., und B. Rowan. 1977. Institutionalized Organizations: Formal Structure as Myth and Ceremony. The American Journal of Sociology 83 (2): 340-363.

Meyer, J. W., G. Krücken, G. S. Drori. 2009. World society: the writings of John W. Meyer. Oxford: Oxford University Press.

Neugebauer, Wolfgang. 2009. Preußen als Kulturstaat. In Das Kultusministerium auf seinen Wirkungsfeldern Schule, Wissenschaft, Kirchen, Künste und Medizinalwesen: Darstellung, Berlin-Brandenburgische Akademie der Wissenschaften unter der Leitung von Wolfgang Neugebauer, Hrsg. Berlin: Akademie.

North, Douglass C. 1990. Institutions, Institutional Change and Economic Performance. Cambridge: Cambridge University Press.

Pinkert, U., und M. Sack, Hrsg. 2014. Theaterpädagogik am Theater. Kontexte und Konzepte von Theatervermittlung. Berlin: Schibri.

Pinto, Alexander. 2013. Freies Theater und das Primat der Stadt: Zum notwendigen Perspektivwechsel in der Theaterdebatte. In Theater entwickeln und planen. Kulturpolitische Konzeptionen zur Reform der Darstellenden Künste, Hrsg. W. Schneider, 243-258. Bielefeld: transcript.

Plank-Baldauf, Christiane., Hrsg. 2019. Praxishandbuch Musiktheater für junges Publikum. Konzepte - Entwicklungen - Herausforderungen. Berlin: Metzler \& Kassel: Bärenreiter.

Primavesi, P. und J. Deck, J., Hrsg. 2014. Stop Teaching! Neue Theaterformen mit Kindern und Jugendlichen. Bielefeld: transcript.

Prinz-Kiesbüye, M.-A., und Y. Schmidt. 2010. Theater für alle, aber nicht von allen? Forum Modernes Theater 25 (1): 45-63.

Reckwitz, Andreas. 2012. Die Erfindung der Kreativität: Zum Prozess gesellschaftlicher Ästhetisierung. Berlin: Suhrkamp.

Reinhardt, Max. 1974. Denkschrift zur Errichtung eines Festspielhaus in Hellbrunn (1917). In Max Reinhardt Schriften: Aufzeichnungen, Briefe, Reden, Hrsg. H. Fetting, 176-182. Berlin: Henschel. 
Roselt, Jens. 2013. Mythos Stadttheater. Vom Weh und Werden einer deutschen Institution. In Theater entwickeln und planen. Kulturpolitische Konzeptionen zur Reform der Darstellenden Künste, Hrsg. W. Schneider, 215-228. Bielefeld: transcript.

Schiller, F. 1995. Die Schaubühne als eine moralische Anstalt betrachtet. In Vom Pathetischen und Erhabenen. Schriften zur Dramentheorie, Hrsg. K. L. Berghahn, 3-13. Stuttgart: Reclam.

Schmidt, Thomas. 2017. Theater, Krise und Reform: Eine Kritik des deutschen Theatersystems. Wiesbaden: Springer Fachmedien.

Scott, W. Richard. 2013. Institutions and Organizations: Ideas and Interests. Los Angeles: Sage.

Seelig, Ludwig. 1914. Geschäftstheater oder Kulturtheater? Berlin: Günther.

Smith, Adam. 2007. An Inquiry into the Nature and Causes of the Wealth of Nations. Amsterdam: MetaLibri Digital Library.

Stauss, S. 2020. A crisis of the singers' market? Shifting discourses on opera from vocal health to changes in the organization of work. Studies in Musical Theatre 14 (2): 207-220.

Toepler, S., and A. Zimmer. 2002. Subsidizing the Arts: Government and the Arts in Western Europe and the United States. In Global culture: media, arts, policy, and globalization, Hrsg. D. Crane, N. Kawashima, und K. Kawasaki, 29-48. New York: Routledge.

Tröndle, M. und R. Behrens, Hrsg. 2018. Beiträge zum Forschungsfeld der Concert Studies. Bielefeld: transcript.

Verhandlungen des Provisorischen Nationalrates des Volksstaates Bayern. 1919. Bd. 1918/19. Stenographischer Bericht über die neunte öffentliche Sitzung, 3. Januar 1919. Bayerische Staatsbibliothek München. https://www.bavarikon.de/object/bav:BSB-MDZ-000 00BSB00009665?lang=de. Zugegriffen: 15. Juni 2020.

Vilar, Jean. 1975. Le T.N.P service public. In Le théâtre service publique, Hrsg. Delcampe A. Paris: Gallimard.

Walgenbach, P., und R. Meyer. 2008. Neoinstitutionalistische Organisationstheorie. Stuttgart: Kohlhammer.

Weber, Max. 2002. Die drei reinen Typen der legitimen Herrschaft: eine soziologische Studie (1922). In Max Weber: Schriften 1894-1922 D. Kaesler (Hrsg.), (717 - 733). Stuttgart: Kröner.

Wild, Christopher J. 2003. Theater der Keuschheit - Keuschheit des Theaters. Eine Geschichte der (Anti-)Theatralität von Gryphius bis Kleist. Freiburg.: Rombach. 
Open Access Dieses Kapitel wird unter der Creative Commons Namensnennung 4.0 International Lizenz (http://creativecommons.org/licenses/by/4.0/deed.de) veröffentlicht, welche die Nutzung, Vervielfältigung, Bearbeitung, Verbreitung und Wiedergabe in jeglichem Medium und Format erlaubt, sofern Sie den/die ursprünglichen Autor(en) und die Quelle ordnungsgemäß nennen, einen Link zur Creative Commons Lizenz beifügen und angeben, ob Änderungen vorgenommen wurden.

Die in diesem Kapitel enthaltenen Bilder und sonstiges Drittmaterial unterliegen ebenfalls der genannten Creative Commons Lizenz, sofern sich aus der Abbildungslegende nichts anderes ergibt. Sofern das betreffende Material nicht unter der genannten Creative Commons Lizenz steht und die betreffende Handlung nicht nach gesetzlichen Vorschriften erlaubt ist, ist für die oben aufgeführten Weiterverwendungen des Materials die Einwilligung des jeweiligen Rechteinhabers einzuholen. 